
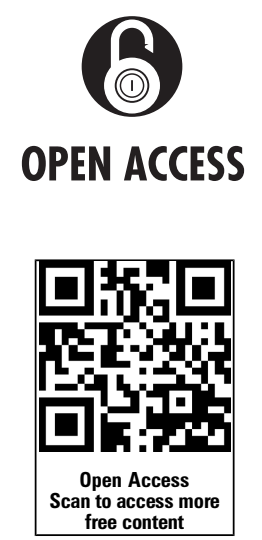

\begin{abstract}
Handling editor Tore K Kvien
- Additional material is

published online only. To view please visit the journal online (http://dx.doi.org/10.1136/ annrheumdis-2014-206405).

${ }^{1}$ Nuffield Department of Medicine, Oxford University, Oxford, UK

${ }^{2}$ Translational Gastroenterology Unit, John Radcliffe Hospital,

Oxford, UK

${ }^{3}$ Sanquin Blood Supply, Division Research and Landsteiner Laboratory, Academic Medical Center, University of Amsterdam, Amsterdam, The Netherlands ${ }^{4}$ Department of Immunology, Churchill Hospital, Oxford University Hospitals NHS Trust, Oxford, UK

${ }^{5}$ Oxford NIHR BRC, Oxford University, Oxford, UK
\end{abstract}

\section{Correspondence to} Professor T Rispens, Sanquin Blood Supply Foundation, Plesmanlaan 125, Amsterdam 1066 CX, The Netherlands; T.Rispens@sanquin.nl

Received 3 August 2014 Revised 5 December 2014 Accepted 13 January 2015 Published Online First 2 February 2015

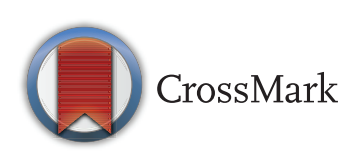

To cite: Culver EL, Vermeulen $\mathrm{E}$, Makuch $\mathrm{M}$, et al. Ann Rheum Dis 2015;74:944-947.

\title{
Increased lgG4 responses to multiple food and animal antigens indicate a polyclonal expansion and differentiation of pre-existing $B$ cells in IgG4-related disease
}

\author{
Emma L Culver, ${ }^{1,2}$ Ellen Vermeulen, ${ }^{3}$ Mateusz Makuch, ${ }^{3}$ Astrid van Leeuwen, ${ }^{3}$ \\ Ross Sadler, ${ }^{4}$ Tamsin Cargill, ${ }^{4}$ Paul Klenerman, ${ }^{1}$ Rob C Aalberse, ${ }^{3}$ \\ S Marieke van Ham, ${ }^{3}$ Eleanor Barnes, ${ }^{1,2,5}$ Theo Rispens $^{3}$
}

\section{ABSTRACT}

Background IgG4-related disease (IgG4-RD) is a systemic fibroinflammatory condition, characterised by an elevated serum IgG4 concentration and abundant lgG4positive plasma cells in the involved organs. An important question is whether the elevated IgG4 response is causal or a reflection of immune-regulatory mechanisms of the disease.

Objectives To investigate if the $\lg G 4$ response in IgG4-RD represents a generalised polyclonal amplification by examining the response to common environmental antigens.

Methods Serum from 24 patients with IgG4-RD (14 treatment-naive, 10 treatment-experienced), 9 patients with primary sclerosing cholangitis and an elevated serum IgG4 (PSC-high IgG4), and 18 healthy controls were tested against egg white and yolk, milk, banana, cat, peanut, rice and wheat antigens by radioimmunoassay.

Results We demonstrated an elevated polyclonal lgG4 response to multiple antigens in patients with IgG4-RD and in PSC-high IgG4, compared with healthy controls. There was a strong correlation between serum IgG4 and antigen-specific responses. Responses to antigens were higher in treatment-naive compared with treatmentexperienced patients with IgG4-RD. Serum electrophoresis and immunofixation demonstrated polyclonality.

Conclusions This is the first study to show enhanced levels of polyclonal IgG4 to multiple antigens in IgG4-RD. This supports that elevated IgG4 levels reflect an aberrant immunological regulation of the overall $\operatorname{lgG} 4$ response, but does not exclude that causality of disease could be antigen-driven.

\section{INTRODUCTION}

IgG4-related disease (IgG4-RD) is a multisystem fibroinflammatory condition, characterised by the development of mass lesions with similar histopathological findings in the involved organs. ${ }^{1}$ Histological characteristics include an infiltrate of lymphocytes and plasma cells, a storiform pattern of fibrosis, obliterative phlebitis and variable presence of eosinophils. An elevated serum IgG4 and abundant accumulation of IgG4-positive plasma cells in affected tissues is frequently seen. An increase in circulating plasmablasts and IgG4 + B cells have also been demonstrated. ${ }^{2}{ }^{3}$ Autoimmune pancreatitis (AIP) and IgG4-related cholangitis (IRC) were the first described manifestations of the disease. ${ }^{4}$

An immune-mediated pathogenesis has been suggested in IgG4-RD, supported by a human leucocyte antigen type II association, presence of autoantibodies and elevated levels of IgG4 and a dramatic response to corticosteroid therapy. ${ }^{5} 6$ Antibodies against a range of autoantigens have been proposed including antinuclear antigens, lactoferrin, carbonic anhydrase II and IV, pancreatic secretory inhibitor and trypsinogens. ${ }^{7} 8$ However, none has been consistently found in the disease, and the fact that they are of the IgG1 and not IgG4 subclass makes their overall significance unclear. A role for Helicobacter pylori plasminogen-binding peptide, through a process of antibody crossreactivity with ubiquitin-protein ligase E3 component $\mathrm{n}$-recognin 2 (molecular mimicry) in genetically predisposed individuals, has been suggested in AIP. ${ }^{9}$ Furthermore, next-generation sequencing of whole blood in patients with IRC highlighted highly abundant IgG4-positive clones in the B cell repertoire, suggesting that specific $B$ cell responses are pivotal to disease pathogenesis. ${ }^{10}$

Our alternative hypothesis is that the elevated IgG4 may not be (primarily) triggered by specific (auto)-antigens, but be an indirect consequence of the expansion of pre-existing IgG4-switched B cells as being responsible for IgG4-RD. In this case, one would expect to find a more generalised and (compared with an antibody response derived from long-lived, bone-marrow-resident plasma cells) a more transient increase in IgG4 antibodies against different antigens that are known to elicit an IgG4 antibody response in the general population. We tested this hypothesis by investigating the level and persistence of the IgG4 response to a variety of known IgG4-inducing non-infectious environmental antigens. We analysed patients with IgG4-RD, patients with primary sclerosing cholangitis (PSC) and elevated IgG4 (a subset of patients with PSC who have an elevated serum IgG4 level but no histological or radiological evidence of IgG4-RD), and healthy controls in a UK cohort. 


\section{METHODS}

Detailed description of patient inclusion criteria and methodology can be found in the online supplementary repository. Antigen-specific IgG4 responses to egg, milk, peanut, banana, rice, wheat and cat were quantified using a previously developed radioimmunoassay. ${ }^{11}$

\section{RESULTS}

\section{Characteristics of the cohort}

Demographics, clinical characteristics and serum immunoglobulin values of patients and controls are shown in the online supplementary table S1. Most patients with IgG4-RD (83\%) had pancreatic (AIP) and/or biliary (IRC) involvement, with 71\% having other systemic organ involvement. The concentration of serum total IgG, IgG4, IgE and, to a lesser extent IgG1, was higher in patients with IgG4-RD versus healthy controls, as well as in the patients with PSC-high IgG4 versus healthy controls (see online supplementary figure S1).

\section{Antigen-specific responses}

We analysed the IgG4 response to proteins from egg, milk, peanut, banana, rice, wheat and cat. In line with our hypothesis, the response to egg $(p=0.004)$, milk $(p=0.04)$, peanut $(\mathrm{p}=0.0003)$, cat dander and serum $(\mathrm{p}=0.012)$, rice and wheat (0.006) antigens was found to be higher in patients with IgG4-RD than in healthy controls, and the response to egg $(p=0.03)$, cat dander and serum $(p=0.04)$, and rice and wheat $(p=0.01)$ antigens was higher in patients with PSC-high IgG4 than in healthy controls (figure 1).

We further examined the correlation of serum immunoglobulin levels with antigen-specific responses. In patients with IgG4-RD, there was a positive correlation between serum IgG4 levels and IgG4 responses to banana (Rank 0.38, 95\% CI -0.002 to $0.67, \mathrm{p}=0.045$ ), peanut (Rank $0.49,95 \%$ CI 0.14 to $0.74, \mathrm{p}=0.007$ ), cat (Rank $0.61,95 \%$ CI 0.29 to 0.80 , $\mathrm{p}=0.0006$ ), rice and wheat (Rank $0.38,95 \%$ CI -0.002 to $0.67, p=0.045$ ) antigens (see online supplementary figure S2). By contrast, total serum IgE levels did not correlate with antigen-specific responses (data not shown).

\section{Treatment-naive and treatment-experienced patients with IgG4-RD}

We also tested the differences in immunoglobulin levels and IgG4-specific antigen responses in 14 treatment-naive and 10 treatment-experienced patients with IgG4-RD receiving corticosteroid therapy. Levels of antibodies to banana $(p=0.001)$, egg $(p=0.039)$, peanut $(p=0.003)$ and cat $(p=0.006)$ antigens were lower in treatment-experienced compared with treatment-naive patients (figure 2), as were serum total $\operatorname{IgG}(p=0.017)$ and IgG4 $(p=0.001)$ (see online supplementary figure S3).

\section{Serum electrophoresis}

We analysed serum electrophoresis data for evidence of monoclonality in patients and controls, but no monoclonal bands in the polyclonal gamma region were observed (see online supplementary figure S4). All individuals showed polyclonality; 11/14 (78.6\%) patients with IgG4-RD, 3/9 (33.3\%) PSC-high patients with IgG4, and 0/18 healthy controls showed hypergammaglobulinaemia. Further immunofixation analysis of patient samples with a diffuse increase in immunoglobulins, using $\operatorname{IgG}$, IgA, IgM, kappa and lambda light-chain antisera, confirmed no monoclonal bands in the gamma region (see online supplementary figure S4).

\section{DISCUSSION}

Our data show that IgG4-RD is associated with an elevated IgG4 response to diverse antigens. Therefore, elevated levels of IgG4 in patients with IgG4-RD may be the result primarily of a polyclonal expansion of many IgG4 B cells irrespective of their specificity. This would imply that factors other than antigen induce an expansion and differentiation of IgG4 B cells, but does not exclude the possibility that a single antigen (self or exogenous) serves as the initial trigger of disease. Antigen-independent involvement of many IgG4-switched B cells implies differential selection via signals that uniquely act on IgG4 B cells. We have recently demonstrated differences in phenotype between IgG1 and IgG4 B cells, ${ }^{12}$ and future studies may reveal traits that uniquely link IgG4 B cells to proliferation in inflamed or fibrotic tissue. ${ }^{13}$ The IgG4-switched B cells may be selectively triggered to expand and differentiate into plasma cells. This may involve interleukin (IL)-21 driving proliferation and expansion of IgG4-switched cells and the upregulation of activation-induced cytidine deaminase, B-lymphocyte-induced maturation protein 1 and Xbox protein 1 , all of which have been shown to be upregulated in patients with various organ manifestations of IgG4-RD. ${ }^{14}{ }^{15}$ Alternatively, signals from the inflamed/fibrotic tissue, including cytokines such as IL-4/IL-13 or IL-10 could selectively stimulate B cells to proliferate into IgG4 plasma cells, either directly or via Th2 and T regulatory cells. ${ }^{16} 17$ Such an environment may be actively sustained via signals from IgG4 B cells themselves, possibly involving IL10. ${ }^{3}$ Furthermore, since IgG4 responses are associated with chronic stimulation, this B cell subset may be more susceptible to expansion due to continued exposure to antigen(s), and therefore there does not need to be a functional link between antibody specificity and potential autoantigen. Importantly, these scenarios are not mutually exclusive. However, it remains unclear if the elevated IgG4 reflects a causal event or epiphenomena. Further research is necessary to determine if the large amounts of IgG4 plasma cells are predominantly the result of disease-associated class switch or disease-associated expansion and terminal differentiation of IgG4 B cells.

The fall in IgG4-specific antigen responses after corticosteroids may be explained by suppressed proliferation of expanded IgG4-switched B cells or suppressed differentiation into IgG4 plasmablasts. Corticosteroids interfere with the production of cytokines critical for $\mathrm{T}$ cell proliferation and interaction, and the binding of interleukins to B cells suppressing proliferation and antibody production. The steroid effect highlights the rapid turnover of the IgG4-producing plasma cells, as opposed to production by long-lived plasma cells in the bone marrow.

Evidence for polyclonality in IgG4-RD is supported by the diversity of antigen specificity, and by serum electrophoresis and immunofixation analysis no monoclonal bands in the polyclonal gamma region was observed (see online supplementary figure S4). However, this is complicated by the ability of the bispecific IgG4 antibody to undergo Fab-arm exchange (known to take place in vivo ${ }^{12}$ ), which limits the potential to detect an oligoclonal response where multiple clones have comparable abundance. In model systems using chimeric antibodies, Fab arm exchange can affect the electrophoretic mobility of IgG4, although if $>50 \%$ of the IgG4 would be monoclonal then $>25 \%$ would still remain monoclonal after Fab-arm exchange. Recently, oligoclonal somatically hypermutated plasmablast populations were demonstrated by next-generation sequencing in patients with active IgG4-RD. ${ }^{19}$ 

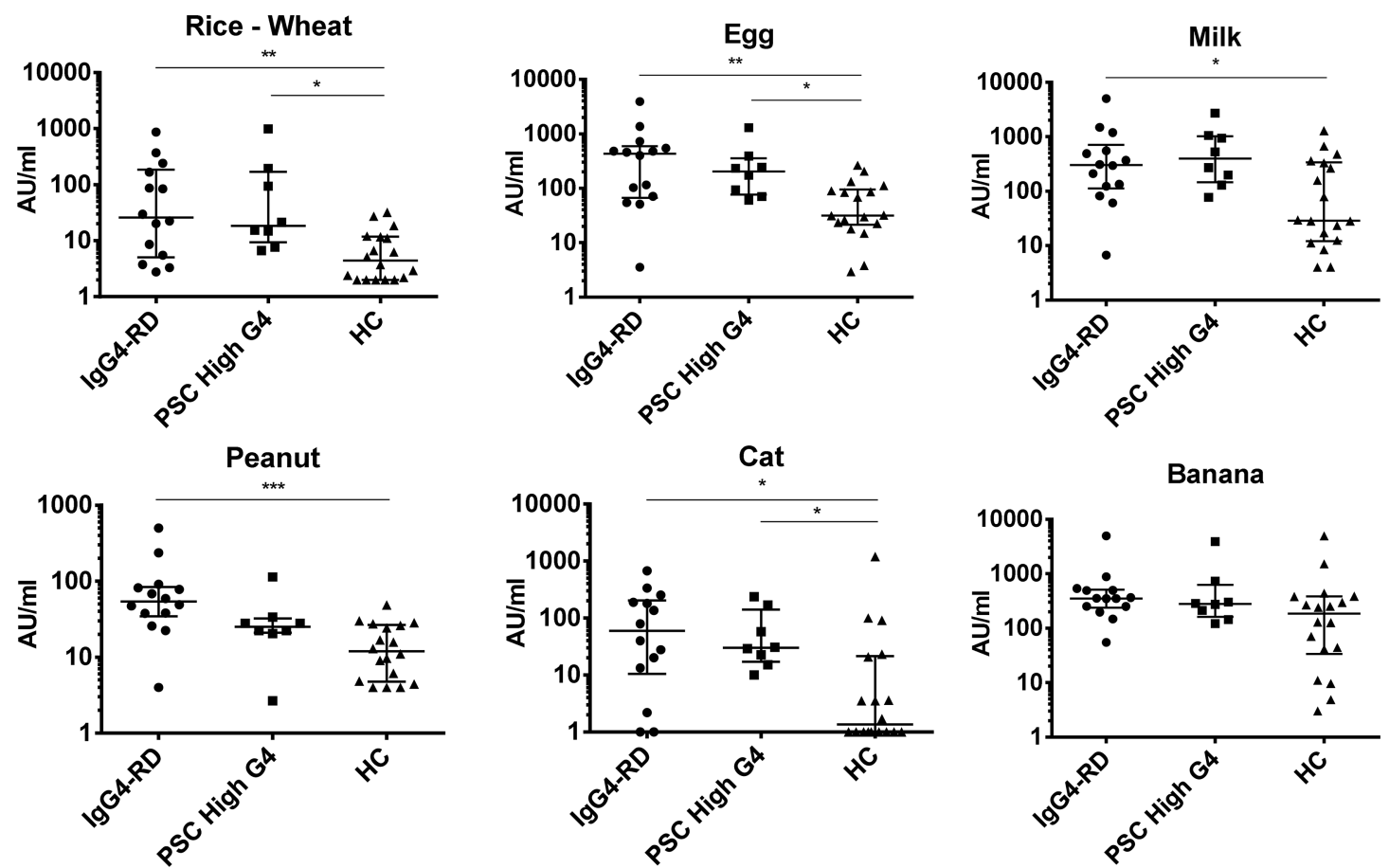

Figure 1 The dot plots show IgG4 antigen-specific responses in treatment-naive patients with IgG4-RD, patients with PSC-high IgG4, and healthy controls. X-axis labels as shown in the figure. Antigens were rice and wheat, egg, milk, peanut, cat dander and serum, and banana. On the $Y$-axis is IgG4-specific antigen response, $\log 10$ scale, in arbitrary units per $\mathrm{mL}$. Error bars represent median and IQR; $p$ values: ${ }^{*} p<0.05,{ }^{*} p<0.005$,

${ }^{* * *} \mathrm{p}<0.001$. HC, healthy controls; IgG4-RD, IgG4-related disease; PSC, primary sclerosing cholangitis.
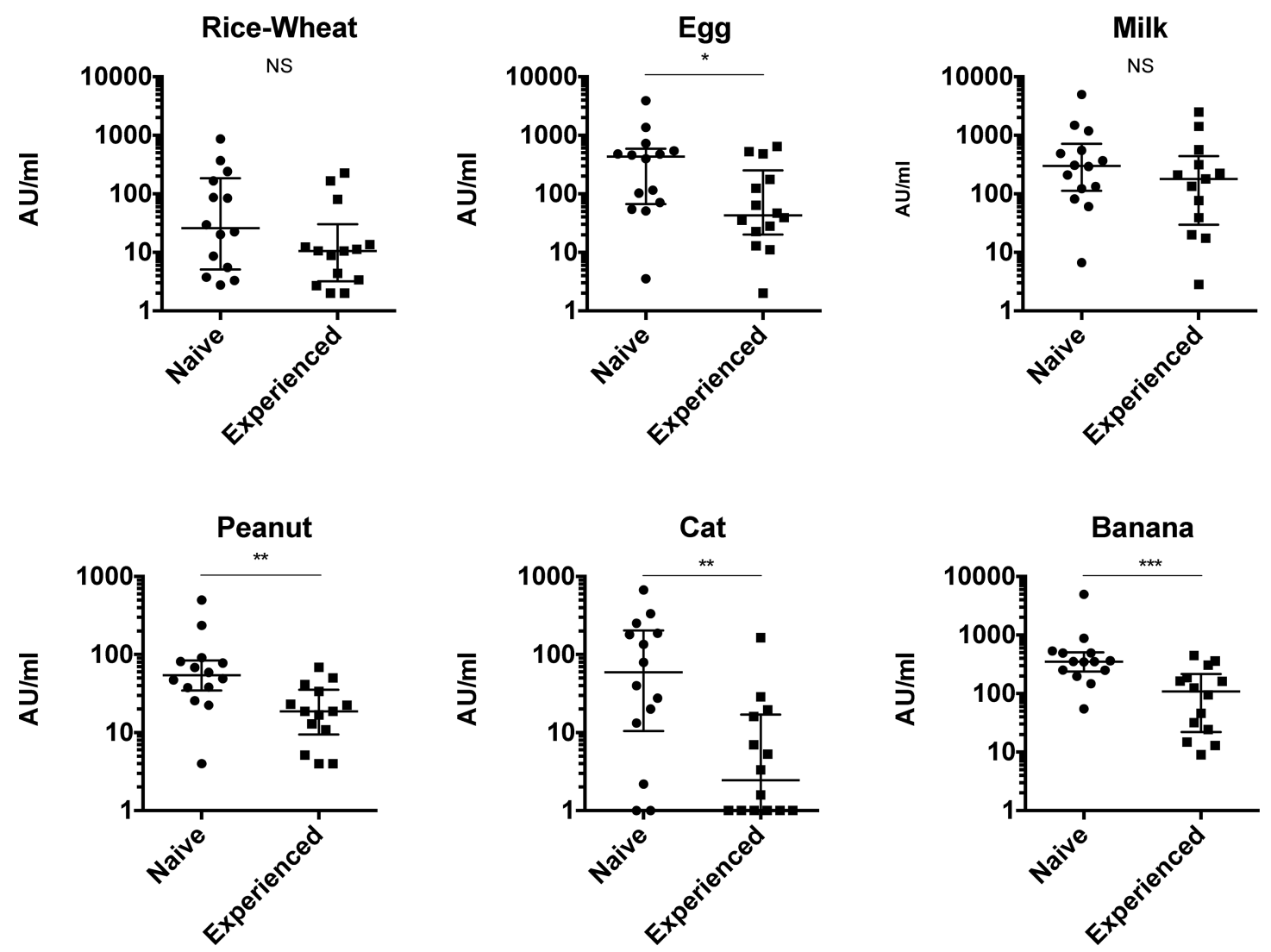

Figure 2 The dot plots show the lgG4 antigen-specific responses in treatment-naive and treatment-experienced (on corticosteroids) patients with IgG4-related disease (IgG4-RD). Antigens were rice and wheat, egg, milk, peanut, cat dander and serum, and banana. Units as in figure 1. Error bars represent median and IQR. Mann-Whitney $p$ values: ${ }^{*} p<0.05,{ }^{* *} p<0.005,{ }^{* *} p<0.001$. 
Mechanisms responsible for driving IgG4-RD (eg, Th2 cytokines) may also increase IgE B cell expansion in certain individuals. In this instance, one may expect an increased predisposition to allergies in later years of life. However, the frequency of allergy/atopy was similar in patient and control groups (see online supplementary table S1). Furthermore, there was no significant difference between total and antigenspecific IgG4 responses in patient and control groups (not shown). However, serum IgE levels in patients with IgG4-RD with allergy/atopy was higher than in those without $(p=0.0255)$ (see online supplementary figure S3); consistent with Della Torre et $a l^{20}$ Therefore, it is plausible that elevated serum IgG4, resulting from polyclonal expansion of many IgG4 B cells irrespective of antigen specificity, may be linked to IgE B cell expansion in a subset of allergic/atopic individuals with IgG4-RD.

To summarise, this is the first study to show an enhanced polyclonal IgG4 response to multiple non-infectious environmental antigens in IgG4-RD. Multiple different antigen responses are higher in treatment-naive compared with treatment-experienced patients. The elevated IgG4 levels may reflect the aberrant immunological regulation of the overall IgG4 response in the disease.

Acknowledgements The authors thank Faye Highfield (NIHR portfolio research facilitator in Oxford) for her invaluable contribution to patient recruitment and clinical data collection.

Contributors ELC diagnosed and consented patients, acquired serum samples, was involved in experimental design and analysis, drafted the original manuscript and the final version. EV was involved in experimental design, performed the RAI assay and was involved in analysis. MM was involved in experimental design and analysis, and edited the manuscript. AvL was involved in experimental design, RAI assay development and optimisation, and performed the assay. RS was involved in experimental design, measurement of serum immunoglobulins, electrophoresis and immunofixation, and edited the manuscript. TC was involved in patient data collection and analysis, and edited the manuscript. PK was involved in experimental critique and editing the manuscript. RCA was involved in study design and concept, developed the RAl assay, involved in analysis, and edited the manuscript. EB was involved in experimental critique and editing the manuscript. SMvH was involved in experimental critique and editing the final manuscript. TR was involved in design and concept of the study, analysis, editing the manuscript. All authors approved the final version of the manuscript.

Funding The study is registered on the UK NIHR portfolio as study number 10776 . ELC receives funding from a Wellcome Trust Research Fellowship [095160/Z10/Z]. EB is supported by the MRC as an MRC Senior Clinical Fellow and the OxNIHR BRC. PK is supported by a Wellcome Trust Grant [091663MA].

Competing interests None.

Ethics approval Oxfordshire REC.

Provenance and peer review Not commissioned; externally peer reviewed.

Open Access This is an Open Access article distributed in accordance with the terms of the Creative Commons Attribution (CC BY 4.0) license, which permits others to distribute, remix, adapt and build upon this work, for commercial use, provided the original work is properly cited. See: http://creativecommons.org/ licenses/by/4.0/

\section{REFERENCES}

1 Stone JH, Zen Y, Deshpande V. IgG4-related disease. N Engl I Med 2012;366:539-51.

2 Wallace ZS, Mattoo H, Carruthers M, et al. Plasmablasts as a biomarker for IgG4-related disease, independent of serum IgG4 concentrations. Ann Rheum Dis 2015; 74:190-5.

3 Lighaam LC, Vermeulen E, Bleker Td, et al. Phenotypic differences between IgG4+ and $\lg \mathrm{G} 1+\mathrm{B}$ cells point to distinct regulation of the $\lg \mathrm{G} 4$ response. J Allergy Clin Immunol 2014;133:267-70.

4 Chari ST, Smyrk TC, Levy MJ, et al. Diagnosis of autoimmune pancreatitis: the Mayo Clinic experience. Clin Gastroenterol Hepatol 2006;4:1010-16.

5 Hamano $\mathrm{H}$, Kawa S, Horiuchi A, et al. High serum lgG4 concentrations in patients with sclerosing pancreatitis. N Engl J Med 2001;344:732-8.

6 Carruthers MN, Khosroshahi A, Augustin T, et al. The diagnostic utility of serum IgG4 concentrations in IgG4-related disease. Ann Rheum Dis 2015;74:14-18.

7 Lohr JM, Faissner R, Koczan D, et al. Autoantibodies against the exocrine pancreas in autoimmune pancreatitis: gene and protein expression profiling and immunoassays identify pancreatic enzymes as a major target of the inflammatory process. Am J Gastroenterol 2010;105:2060-71.

8 Asada M, Nishio A, Uchida K, et al. Identification of a novel autoantibody against pancreatic secretory trypsin inhibitor in patients with autoimmune pancreatitis. Pancreas 2006:33:20-6.

9 Frulloni L, Lunardi C, Simone R, et al. Identification of a novel antibody associated with autoimmune pancreatitis. N Engl J Med 2009;361:2135-42.

10 Maillette de Buy Wenniger LJ, Doorenspleet ME, Klarenbeek PL, et al. Immunoglobulin $\mathrm{G} 4+$ clones identified by next-generation sequencing dominate the B cell receptor repertoire in immunoglobulin G4 associated cholangitis. Hepatology 2013:57:2390-8

11 Calkhoven $\mathrm{PG}$, Aalbers M, Koshte VL, et al. Relationship between IgG1 and IgG4 antibodies to foods and the development of IgE antibodies to inhalant allergens. II. Increased levels of IgG antibodies to foods in children who subsequently develop IgE antibodies to inhalant allergens. Clin Exp Allergy 1991;21:91-8.

12 Labrijn AF, Buijsse AO, van den Bremer ET, et al. Therapeutic lgG4 antibodies engage in Fab-arm exchange with endogenous human IgG4 in vivo. Nat Biotechnol 2009:27:767-71.

13 Lighaam LC, Aalberse RC, Rispens T. IgG4-related fibrotic diseases from an immunological perspective: regulators out of control? Int I Rheumatol 2012;2012:789164.

14 Kuchen S, Robbins R, Sims GP, et al. Essential role of IL-21 in B cell activation, expansion, and plasma cell generation during $\mathrm{CD}^{+} \mathrm{T}$ cell-B cell collaboration J Immunol 2007;179:5886-96.

15 Tsuboi H, Matsuo N, lizuka M, et al. Analysis of IgG4 class switch-related molecules in IgG4-related disease. Arthritis Res Ther 2012;14:R171.

16 Jeannin $\mathrm{P}$, Lecoanet $\mathrm{S}$, Delneste $\mathrm{Y}$, et al. IgE versus $\lg \mathrm{G} 4$ production can be differentially regulated by IL-10. J Immunol 1998;160:3555-61.

17 Miyoshi H, Uchida K, Taniguchi T, et al. Circulating naive and CD4+CD25high regulatory T cells in patients with autoimmune pancreatitis. Pancreas 2008;36:133-40.

18 van de Veen W, Stanic B, Yaman G, et al. IgG4 production is confined to human IL-10-producing regulatory $B$ cells that suppress antigen-specific immune responses. J Allergy Clin Immunol. 2013;131:1204-12.

19 Mattoo H, Mahajan VS, Della-Torre E, et al. De novo oligoclonal expansions of circulating plasmablasts in active and relapsing IgG4-related disease. J Allergy Clin Immunol 2014;134:679-87.

20 Della Torre E, Mattoo H, Mahajan VS, et al. Prevalence of atopy, eosinophilia, and IgE elevation in IgG4-related disease. Allergy 2014;69:269-72. 